\title{
Ambos, Claus, Rösch, Petra, Schneidmüller, Bernd, Weinfurter, Stefan, Bild und Ritual. Visuelle Kulturen in historischer Perspektive
}

Pierre Monnet

\section{(2) OpenEdition \\ Journals}

Édition électronique

URL : http://journals.openedition.org/ifha/2107

DOI : 10.4000/ifha. 2107

ISSN : 2198-8943

Éditeur

IFRA - Institut franco-allemand (sciences historiques et sociales)

Référence électronique

Pierre Monnet, « Ambos, Claus, Rösch, Petra, Schneidmüller, Bernd, Weinfurter, Stefan, Bild und Ritual. Visuelle Kulturen in historischer Perspektive », Revue de l'IFHA [En ligne], Date de recension, mis en ligne le 01 janvier 2010, consulté le 22 septembre 2020. URL : http://journals.openedition.org/ifha/2107 ; DOI : https://doi.org/10.4000/ifha.2107

Ce document a été généré automatiquement le 22 septembre 2020.

(CIFHA 


\title{
Ambos, Claus, Rösch, Petra, Schneidmüller, Bernd, Weinfurter, Stefan, Bild und Ritual. Visuelle Kulturen in historischer Perspektive
}

\author{
Pierre Monnet
}

1 Ce recueil d'études rassemble des communications élaborées au sein d'un programme transversal de recherche (Sonderforschungsbereich) établi depuis 2002 à l'Université de Heidelberg sous le titre « La dynamique des rituels : processus socioculturels dans une perspective historique et culturelle comparée ». Le mot qui compte dans cette enquête, et dans ce livre, est bien celui de dynamique, censé corriger la dérive toujours possible d'une histoire figée et mécanique des rituels. Pour le prouver, le choix a été fait de soumettre les images à cette dynamique des rituels en plongeant celle-ci dans une analyse, somme toute classique, à double entrée : les images dans les rituels et les rituels dans les images. Si le croisement entre rituels et images n'est pas neuf, l'originalité tient ici à l'ampleur du balayage thématique, chronologique et géographique réalisé, passant de la Méditerranée antique à l'Occident médiéval latin puis à l'Inde et à la Chine, en interrogeant chaque fois aussi bien la signification et la fonction du visuel dans le rituel grâce à l'image, que les stratégies narratives et symboliques portées par les rituels dans les images. On sent bien que l'un des enjeux de la réflexion réside moins dans un inventaire des images entourant ou restituant le rituel que de savoir dans quel contexte social et culturel ce qui est appelé une "image " (question importante) est investi de la mission de rendre visible ou efficace tel ou tel rituel. Ce déplacement de la question conduit ainsi les porteurs du projet à convoquer plusieurs disciplines (histoire, histoire de l'art, ethnologie, théologie, anthropologie) et à multiplier les supports et objets illustrés : livres, fresques, gravures, papyri, stèles, monnaies, autels...

2 La première partie de l'ouvrage traite donc de la fonction des images dans les rituels, essentiellement sous l'angle (mais il y en a d'autres qu'il eût au moins fallu recenser et 
formuler) de l'influence exercée par l'image sur le rituel, et ce dans la quadruple perspective de la consécration des images, de leur efficacité dans les rituels, de leur fonctionnalité et de la remise en cause dont elles peuvent faire l'objet. L'image s'y trouve investie d'une efficacité qui accentue la divinité de tel Dieu, qu'il soit égyptien, mésopotamien ou bouddhiste, et partant augmente le statut et le pouvoir de son médiateur, particulièrement en terme de protection et de souveraineté pour le roi ou empereur qui parvient à en manier la logique à son profit. Ce sont ici les démonstrations qui intéressent le plus, ainsi que la documentation mobilisée, davantage que l'hypothèse elle-même déjà plusieurs fois formulée et explorée. De ce point de vue, un chapeau conclusif, plus encore qu'introductif, aurait dû véritablement comparer les exemples convoqués, pour faire le bilan des possibilités et des limites d'un grand écart certes contrôlé et productif, mais qui n'en repose pas moins sur une interrogation portant à la fois sur Babylone, l'Égypte pharaonique, la Chine du VIe siècle, le culte des bustes impériaux romains et celui des rois mages aux XIIe et XIIIe siècles. Le dernier chapitre de ce premier mouvement consacré aux images dans les rituels se révèle le plus intéressant, et sans doute celui pour lequel la comparaison à large rayon chronologique et géographique apparaît la plus productive. Il traite en effet de la critique envers l'image quand elle est couplée aux rituels, aboutissant selon les cas (mais dans quels contextes convergents ou divergents ?) à une destruction à la mesure de la loyauté et de la conformité que les images étaient censées conférer aux rituels.

La seconde partie du recueil traite des images des rituels sous l'angle de leur production (essentiellement dans une tension entre le texte et l'image), de leur force de représentation symbolique et de la dynamique, rebaptisée "interactivité visuelle ", dont elles sont porteuses. En posant la question de savoir quelles histoires et quelles configurations de rituels sont rapportées par l'image, c'est paradoxalement moins la question de l'interprétation des images mêmes que celle des rituels qui est finalement posée. Quels sont en effet les rituels visualisables, et quels sont ceux qui ne le sont pas ? Et, parmi les premiers, qu'apporte de plus l'image à la compréhension du rituel, non seulement par les contemporains mais aussi par les historiens bien plus tard (un double niveau qu'il eût fallu sans doute mieux articuler, à l'instar de ce que fait K. Oschema (p. 160-171) dans sa contribution sur les images du meurtre probable d'Edouard II d'Angleterre en 1327, dont les représentations renvoient de tout autres caractères rituels que les chroniques, une distance souvent mal maîtrisée ou décodée non par les médiévaux mais par les médiévistes) ? On ne peut s'empêcher de penser que les réflexions poursuivies tout au long de cette seconde partie se révèlent plus riches et complexes que celles formulées au cours de la première. On plonge en effet ici au cœur de la logique de fonctionnement des modalités visuelles de plusieurs types d'actions rituelles, qu'il s'agisse du couronnement du roi des Romains mobilisant et adaptant une tradition du couronnement de la Vierge ou bien des représentations des rituels de la Bulle d'Or de 1356 un peu moins d'un siècle et demi plus tard (1485) qui montrent combien l'Empire entretemps a trouvé un ordre territorial et spatial dont on charge la Bulle d'être le reflet (B. Schneidmüller, p. 210-230). Même les confrontations avec le lointain paraissent ici plus justifiées, ainsi que celle avec le rituel chinois de l'empereur à la charrue (L. Ledderose, p. 185-196) particulièrement apprécié sous la dynastie des Qing (1644-1911) et rapporté ici dans un kaléidoscope d'images et de récits provenant tant de la cour chinoise même que des Jésuites résidant en Chine puis des physiocrates européens qui en ont adapté la relation. L'homogénéité plus grande de cette seconde partie tient selon nous au choix d'une série de concepts et d'échelles communs, pour 
l'essentiel des images de rituels au niveau royal et impérial sous l'angle de l'ordre spatial.

4 À défaut de conclusion, qui à notre avis eût été bienvenue, une réflexion finale et parfois déconnectée du propos central de l'ouvrage interroge l'avenir, les chances, les impasses et les perspectives d'une science historique et culturelle des images à l'ère d'une multimédialisation envahissante de notre quotidien, laquelle de fait confère au statut et aux fonctions de l'image un nouveau caractère, et à leur réception une nouvelle dimension.

5 Le recueil, contribution importante à la problématique de la fonction de l'image dans le contexte des rituels ouverte en 1990 par Hans Belting dans Bild und Kult, se signale donc par une originalité certaine quoique disparate des contributions et par le pari de l'innovation surgie d'une confrontation entre aires et époques diverses. La question demeure cependant de savoir si, en dehors de l'Occident médiéval chrétien et latin qui inspire majoritairement la réflexion des porteurs scientifiques de cette enquête, les constructions épistémologiques portant sur l'efficacité des rituels et la culture visuelle peuvent être reportées, même avec mille précautions, sur des contextes aussi lointains et étrangers que l'Inde ou la Chine.

6 Pierre Monnet (EHESS, Paris) 